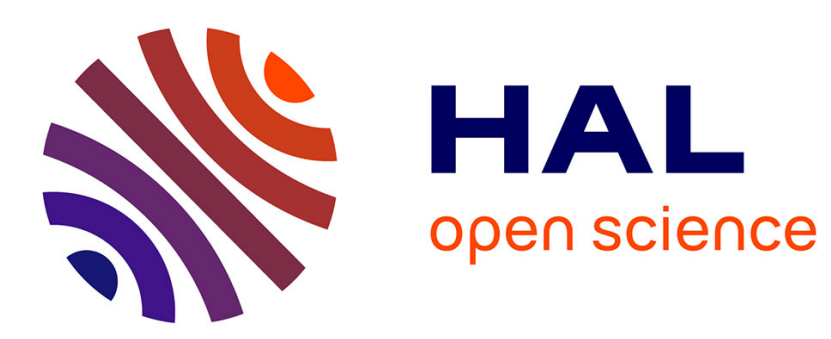

\title{
Motion of Viscous Droplets in Rough Confinement: Paradoxical Lubrication
}

Ludovic Keiser, Armelle Keiser, Manon L'estimé, José Bico, Etienne Reyssat

\section{To cite this version:}

Ludovic Keiser, Armelle Keiser, Manon L'estimé, José Bico, Etienne Reyssat. Motion of Viscous Droplets in Rough Confinement: Paradoxical Lubrication. Physical Review Letters, 2019, 122 (7), 10.1103/PhysRevLett.122.074501 . hal-02347156

\section{HAL Id: hal-02347156 https://hal.science/hal-02347156}

Submitted on 5 Nov 2019

HAL is a multi-disciplinary open access archive for the deposit and dissemination of scientific research documents, whether they are published or not. The documents may come from teaching and research institutions in France or abroad, or from public or private research centers.
L'archive ouverte pluridisciplinaire HAL, est destinée au dépôt et à la diffusion de documents scientifiques de niveau recherche, publiés ou non, émanant des établissements d'enseignement et de recherche français ou étrangers, des laboratoires publics ou privés. 


\title{
Motion of viscous droplets in a rough confinement: a paradoxical lubrication
}

\author{
Ludovic Keiser ${ }^{12},{ }^{*}$ Armelle Keiser ${ }^{1}$, Manon L'Estimé ${ }^{1}$, José Bico ${ }^{1}$, and Étienne Reyssat ${ }^{1 \dagger}$ \\ 1 Laboratoire de Physique et Mécanique des Milieux Hétérogènes (PMMH), \\ Sorbonne Université, 7 quai Saint-Bernard, barre Cassan 75005 Paris, \\ CNRS UMR 7636, ESPCI Paris - PSL Research University, Univ. Paris Diderot and \\ 2 Total S.A., Pôle d'Études et de Recherche de Lacq, BP47 64170 Lacq, France
}

(Dated: November 4, 2019)

\begin{abstract}
We study the sedimentation of highly viscous droplets confined inside Hele-Shaw cells with textured walls of controlled topography. In contrast with common observations on superhydrophobic surfaces, roughness tends here to significantly increase viscous friction, thus substantially decreasing the droplets mobility. However, reducing confinement induces a jump in the velocity as droplets can slide on a lubricating layer of the surrounding fluid thicker than the roughness features. We demonstrate that increasing the viscosity of the surrounding liquid may counter-intuitively enhance the mobility of a droplet sliding along a rough wall. Similarly, a sharp change of the droplet mobility is observed as the amplitude of the roughness is modified. These results illustrate the non-trivial friction processes at the scale of the roughness, and the coupling between viscous dissipation in the drop, in the front meniscus and in the lubricating film. They could enable to specifically control the speed of droplets or capsules in microchannels, based on their rheological properties.
\end{abstract}

Numerous interfacial instabilities induce the emulsification of petroleum in porous media such as snap-off [1], viscous fingering [2-4] or pure capillary phenomena [5]. The displacement of the generated droplets in the porous network leads to complex rheology [6]. More generally, the transport of droplets in confined environment is crucial for medical and chemical engineering applications, as illustrated by the fast development of digital microfluidics during the last two decades $[7,8]$. This problem motivated theoretical, numerical and experimental works, with the aim of understanding the dynamics of confined drops in ideal geometries [9-17]. Although they demonstrated the major contribution of lubricating films, these studies mainly remained focused on smooth confining walls. In common situations, walls may however present natural or engineered roughness features. $\mathrm{Nu}-$ merous works have also been dedicated to the reduced friction on

superhydrophobic surfaces [18-23] and to the reduced adhesion on liquid-infused surfaces of controlled topography [24-26]. However the impact of roughness on the dynamical properties of the lubricating film has so far not been extensively studied.

In this Letter, we analyze the dynamics of non-wetting confined oil droplets falling in a Hele-Shaw cell filled with a surfactant solution of lower viscosity. By comparing the dynamics of droplets moving in smooth and rough confinements, we propose to highlight the impact of roughness on the mobility of the droplets.

Our experimental setup (Fig. 1) is composed of two parallel glass plates covered with a square array of micropillars of square cross-section (height $h$, width $w$, separation $s$ ) cast in a layer of transparent resin (NOA from Thorlabs, Inc.) using photolithography techniques (see details in the Supplementary Information). The walls are separated by a gap $e$ ranging from 300 to $1500 \mu \mathrm{m}$, much larger than the micrometric height $h$ of the textures, as

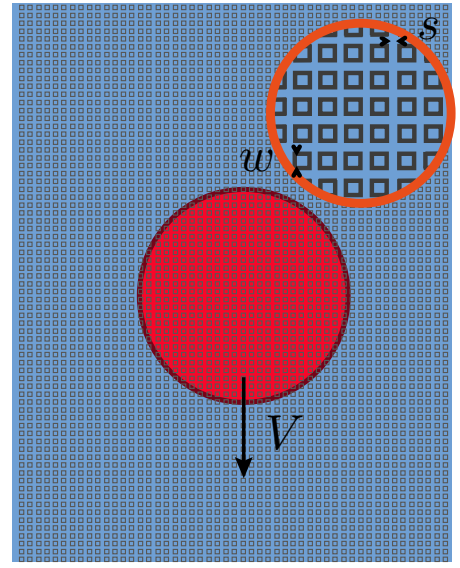

Front view

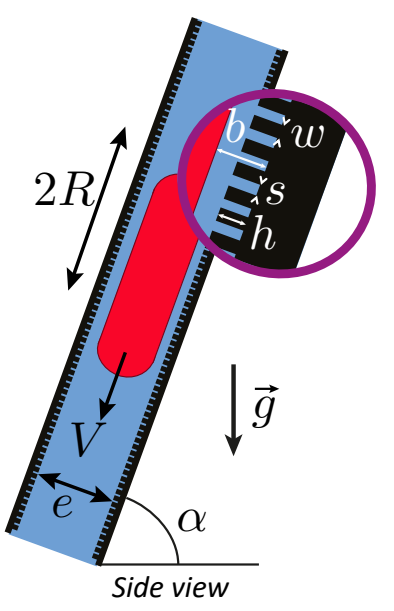

FIG. 1: Experimental setup. A drop of fluorinated oil (in red) is confined between the walls of a rough Hele-Shaw cell filled with a surfactant solution (in blue). The cell is inclined by an angle $\alpha$, with respect to the horizontal. The thickness $e$ of the gap ranges from 300 to $1500 \mu \mathrm{m}$. The cell walls are covered with a lattice of micropillars of square cross-section of height $h \ll e$, width $w$ and separation $s$. The denser drop (radius $R$ and volume $\Omega=\pi R^{2} e$ ) falls under gravity with a steady velocity $V$. A lubricating film of surfactant solution separates the drop from the walls, with an average thickness $b$ between the drop and the bottom of the roughness.

sketched in Fig. 1. The cell is immersed vertically in a bath of a surfactant solution, containing $5 \%$ in mass of a commercial soap (PAIC, from Colgate-Palmolive), of dynamic viscosity $\mu_{w}=1 \mathrm{mPa}$.s. Drops of fluorinated silicone oil (poly(3,3,3)-trifluoropropylmethylsiloxane, from Gelest, Inc.) of volume $\Omega$ and viscosity $\mu_{o}$ ranging from 180 to $18000 \mathrm{mPa}$.s are inserted in the upper part of the cell. The interfacial tension between both liquids $\gamma=5-6 \mathrm{mN} / \mathrm{m}$ (depending on the oil viscosity) is measured with the pendant drop method. The oil density 

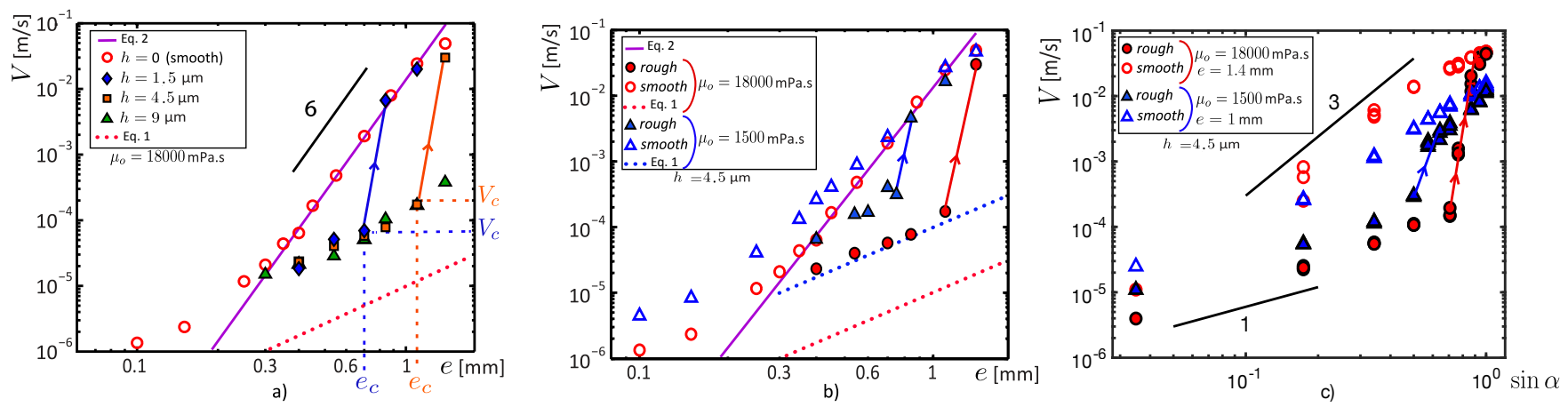

FIG. 2: a) Falling velocity $V$ in vertical cell vs. the gap of the cell $e$ for different pillar heights $h$, for $\mu_{o}=18000 \mathrm{mPa}$.s, texture width $w=180 \mu \mathrm{m}$ and spacing $s=35 \mu \mathrm{m}$. For smooth walls (empty red circles), $V$ increases as $e^{6}$, as described by Eq. 2 (solid magenta line). In rough cells and below a critical velocity $V_{c}, V$ grows as $e^{2}$ and is significantly smaller than in smooth cells. Velocities are one order of magnitude larger than expected from a basic Poiseuille flow (dotted red line), a signature of the slip introduced by the roughness. For $e>e_{c}$, or equivalently $V>V_{c}$, a "jump" of velocity is observed (indicated by arrows and illustrated in the Supplementary Movie 1) and $V$ is captured by Eq. 2, as for smooth cells. The critical velocity $V_{c}$ increases as the textures height $h$ increases, and for $h=9 \mu \mathrm{m}$ (green triangles), we do not observe any jump in velocity within the explored gaps. b) Influence of oil viscosity on drop dynamics in a vertical cell. In smooth cells, the velocity barely depends on $\mu_{o}$ (as described by Eq. 2). Conversely in rough cells and below $V_{c}$, the velocity decreases as $\mu_{o}$ increases, reflecting that viscous dissipation is localized inside the drop in the "weakly lubricated" regime. c) Falling velocity in Hele-Shaw cells tilted by an angle $\alpha$ with respect to the horizontal, for two configurations $\left(e, \mu_{o}\right)$ : $(1 \mathrm{~mm}, 1500 \mathrm{mPa} . \mathrm{s})$ and $(1.4 \mathrm{~mm}, 18000 \mathrm{mPa} . \mathrm{s})$. A sharp velocity transition is also observed as $\alpha$ exceeds a critical value. The drops keep falling even at the lowest inclinations $\left(\alpha=2.5^{\circ}\right)$, highlighting the absence of pinning of the drop at the top of the pillars, which would result in a vanishing velocity at a finite value of the angle $\alpha$.

$\rho_{o}=1230-1250 \mathrm{~kg} / \mathrm{m}^{3}$ is larger than the density of water $\left(\rho_{w}=997 \mathrm{~kg} / \mathrm{m}^{3}\right)$ so that drops fall under gravity. This simple configuration ensures a precisely controlled driving force, enabling a thorough characterization of the dissipation mechanisms $[14,16]$. As the surfactant solution completely wets the surface of the cell walls, no adhesion hinders the motion of the oil drops. The vertical trajectory of the drops is monitored with a digital camera. The drops are observed to reach a constant velocity $V$ after a few millimeters of descent. For fixed values of $\mu_{w}, \mu_{o}$ and $e, V$ is independent of the volume within the limit of confined drops $(R>e)$. Due to the high contrast in viscosity between the drops and the surrounding solution, we expect the motion of the drops to markedly rely on the thickness of the lubricating film $b$, as shown by recent studies $[14,16]$.

In a cell with smooth walls, $b$ follows the classical Bretherton's law [27] $b \sim e / 2 C a^{2 / 3}$, for capillary numbers $C a=\mu_{w} V / \gamma$ ranging from $10^{-5}$ to $10^{-2}$, as confirmed by recent experimental investigations $[11,17]$. In this configuration, the dimensionless parameter $m=$ $\mu_{w} e / \mu_{o} b$ characterizes the efficiency of the lubrication process. For large values of $m$, the films of water are too thin to lubricate efficiently the motion. The flow in the volume of the drop thus follows a classical Poiseuille profile, which corresponds to the friction force $F_{f}=$ $12 \pi \mu_{o} V R^{2} / e$. Balancing $F_{f}$ with the apparent weight of the "pancake" drop in water $F_{g}=\pi\left(\rho_{o}-\rho_{w}\right) g R^{2} e$ leads to the sedimentation velocity:

$$
V=\frac{\left(\rho_{o}-\rho_{w}\right) g \sin \alpha}{12 \mu_{o}} e^{2}
$$

However in the present experimental conditions, the large inner-to-outer viscosity ratio $\left(\mu_{o} / \mu_{w}>180\right)$ favors the opposite limit $m \ll 1$. In this regime, the drop tends to slide on the water films as a solid. Viscous dissipation is now localized in the lubricating film, which results in the sublinear friction force $F_{f} \sim \mu_{w} V R^{2} / b \sim \gamma R^{2} C a^{1 / 3} / e$. and leads to a strong dependence of the velocity on the gap:

$$
V=\beta \frac{\left(\rho_{o}-\rho_{w}\right)^{3} g^{3} \sin ^{3} \alpha}{\mu_{w} \gamma^{2}} e^{6},
$$

where $\beta=4.3 \cdot 10^{-2}$ is a numerical factor experimentally determined in a previous work [16]. This regime, represented by empty circles and full lines in Fig. $2 \mathrm{a}$ and $2 \mathrm{~b}$, is hereafter called "strongly lubricated" and corresponds to velocities independent of the drop viscosity $\mu_{o}$ (within the limit $m \ll 1)$.

In a cell with rough walls, the dynamics is dramatically altered, and the velocity is strongly reduced, as shown in Fig. 2. Although $V$ displays a square dependence with $e$, its magnitude is about ten times larger than expected from a basic Poiseuille flow in the drop (Eq. 1 and dotted lines in Fig. 2). We interpret this shift as a consequence of an effective slip due to the presence of water atop and between the pillars $[19,21]$. We refer 


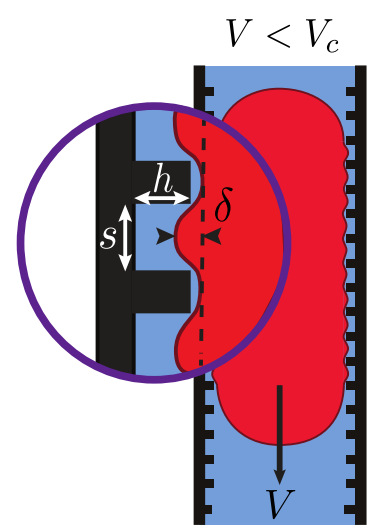

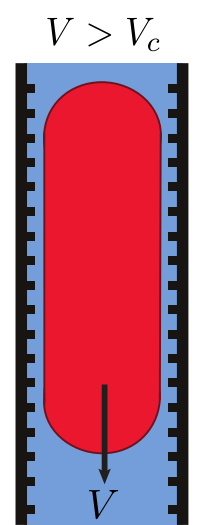

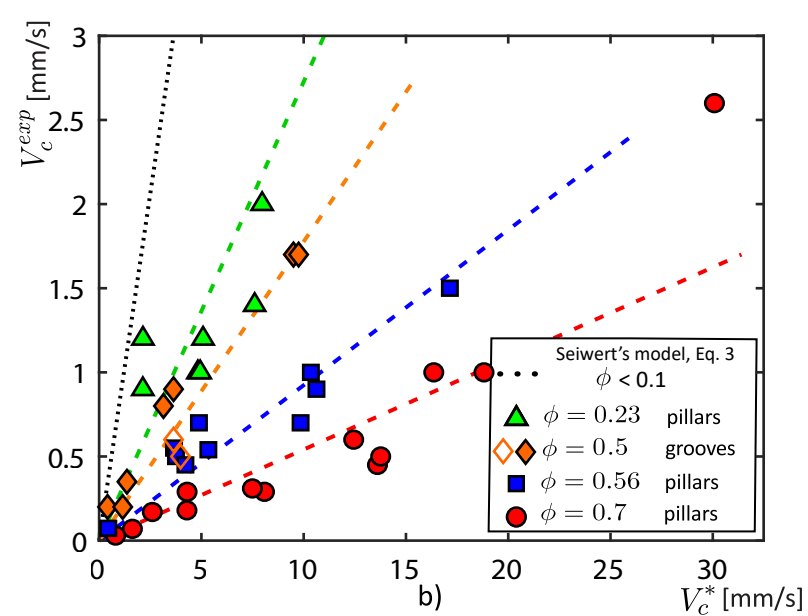

FIG. 3: a) At small velocity $\left(V<V_{c}\right)$, the roughness-induced reduction of the viscous stress at the front meniscus of the drop hinders the deformation of the interface and the deposition of a lubricating film. The drop is compressed in the pillars by the capillary pressure and its interface is corrugated. Furthermore, a thin nanometric film of water, stabilized by disjoining pressure, isolates the drop from the top of the pillars. In this configuration, viscous dissipation localizes in the viscous drop, which strongly reduces its mobility. At large velocity $\left(V>V_{c}\right)$, the viscous stress at the front meniscus is strong enough to deform the interface and to deposit a film where viscous dissipation localizes. The dynamics is thus similar to the case of smooth confinement. b) Experimental critical velocity as a function of $V_{c}^{*}$ (Eq. 3), for different oil viscosities $\mu_{o}$ and pillars heights $h$, for various pillars densities and geometries: $\phi=0.23$ (green triangles, pillars), $\phi=0.50$ (full/empty orange diamonds, grooves perpendicular/parallel to the direction of motion of the droplets), $\phi=0.56$ (blue squares, pillars), $\phi=0.70$ (red circles, pillars). The large variations of $V_{c}$ mainly originate from variations of $h$, ranging from 1.5 to $9 \mu \mathrm{m}$. The variations of $\mu_{o}$ from 180 to $18000 \mathrm{mPa}$.s only slightly alters $V_{c}$, due to the change of $e_{c}$, as shown in Fig.2.

to this regime as "weakly lubricated", and discuss hereafter its origin. Above a critical gap thickness $e_{c}$, corresponding to a critical speed $V_{c}$, we observe an abrupt jump in velocity, represented by arrows in Fig. 2 (see also the Supplemental Movie 1). For $e>e_{c}$, the measured velocities are similar to the velocities obtained in a smooth cell. The "strongly lubricated" regime is thus recovered and the velocity increases by more than two orders of magnitude at the jump. We carried out experiments with various texture heights $h$ for a given pattern $(w=200 \mu \mathrm{m}, s=25 \mu \mathrm{m})$. Fig. 2a illustrates that as $h$ is increased from 1.5 to $9 \mu \mathrm{m}$, the critical gap and critical velocity also increase. For the largest height $(h=9 \mu \mathrm{m})$, the critical velocity $V_{c}$ is even not reached within the explored range of parameters. This spectacular effect is also observed for droplets of smaller viscosity, as plotted in Fig. 2b. In the "weakly lubricated" regime $\left(V<V_{c}\right)$, the velocity of the drops increases as the oil viscosity $\mu_{o}$ is decreased (from 18000 to $1500 \mathrm{mPa} . \mathrm{s}$ ), which reflects that viscous dissipation is localized in the volume of the drop. For $\mu_{o}=1500 \mathrm{mPa} . \mathrm{s}$ (blue triangles) the velocity jump is also observed and for $e>e_{c}$, all velocities collapse on the same curve corresponding to the "strongly lubricated" regime (Eq. 2). The transition is also observed in cells of given thickness but tilted with respect to the horizontal with an angle $\alpha$ (Fig. 2c). At small $\alpha$, weakly lubricated drops move slowly but still smoothly, indicating the absence of pinning on the textures. Increasing effective gravity by tilting the cell, one recovers the sharp transition to the high velocity regime where drops do not feel the roughness. For a given texture pattern, the critical velocity $V_{c}$ is the same as the one measured in experiments where the gap has been varied.

The massive reduction of the mobility of non-wetting drops with the wall roughness strongly contrasts with the common behavior of water confined in superhydrophobic channels, where the roughness-induced slippage at the walls promotes higher mobility in comparison with smooth walls [18-23]. We interpret this paradox as a consequence of the role of the surrounding fluid as a lubricant. Analogous results were observed by Seiwert et al. in a recent study on the coating of a textured plate removed from a bath of liquid [28]. Below a critical withdrawal velocity $V_{c}$, the liquid invades the textures (a dilute array of pillars), but the authors do not observe any coating film above the height of the pillars. However, a coating film is deposited and Bretherton's law is progressively recovered as the withdrawing velocity is increased above $V_{c}$. A velocity threshold thus appears for the deposition of a film above the textures. When applied to our configuration, this scenario leads to the two regimes sketched in Fig. 3a. For $V<V_{c}$, the surrounding solution only fills the textures. The friction of the droplet on the textured walls is thus complex. Due to molecular interactions, a thin film of the solution may be present on the top of the pillars. The thickness of such a thin film is of the order of tens of nanometers [11, 25, 29]; its lubricating properties are therefore limited [16]. Furthermore, due to the strong confinement, Laplace pressure induces the formation of bumps between pillars of am- 
plitude $\delta \sim s^{2} / e \sim 500 \mathrm{~nm}$, which results into additional friction localized in the viscous drop [21, 30]. The overall dissipation in this "weakly lubricated" regime should thus depend on the topography of the texture and more specifically on the pillar density $\phi=(w /(w+s))^{2}$. Describing in detail these processes would deserve a dedicated study and is beyond the scope of this Letter. In the opposite condition $V>V_{c}$, a lubricating layer of the solution covers the textures and reduces dramatically the friction, as viscous dissipation localizes in the lubricating film of low viscosity. Modelling the impregnated textures as an effective viscous liquid layer in Bretherton's derivation, Seiwert et al. estimate the evolution of the deposited layer in their coating process [28]. As $V$ is increased, the thickness quickly converges to the classical Bretherton's law [28] in agreement with the sudden jump observed in our experiments and with different derivations accounting for wall slip [31, 32]. The transition between both regimes in the experiments of Seiwert is smooth: the thickness varies continuously with the imposed coating velocity. In contrast, our experiment is performed at a controlled driving force, which leads to a discontinuity in the velocity. In terms of scaling, $V_{c}$ can be estimated as the velocity required to obtain a coating film of thickness $h$ in a standard smooth situation [28]:

$$
V_{c}^{*} \sim\left(\frac{h}{e_{c} / 2}\right)^{3 / 2} \frac{\gamma}{\mu_{w}}
$$

However, we also expect the pillar density $\phi$ to play a major role in setting the transition. In Fig. $3 \mathrm{~b}$, we plot the evolution of the critical velocity $V_{c}^{\exp }$ as a function of $V_{c}^{*}$ for different values of $\phi$. Each set of measurements corresponds to different oil viscosities and pillar heights. Although experimental data display some scattering, $V_{c}^{\exp }$ convincingly appears to be proportional to $V_{c}^{*}$, confirming the scaling. In addition, the numerical prefactor is rather low for high values of $\phi$ (of the order of $1 / 20$ for $\phi=0.7)$. In the limit $\phi \rightarrow 1$ corresponding to a smooth wall, we even expect $V_{c}^{\exp }$ to vanish. Conversely, the prefactor gets progressively closer to unity as $\phi$ decreases. The result from Seiwert et al., represented with a dotted line was experimentally obtained within the limit $\phi<0.1$ [28].

The value of $V_{c}^{*}$ is inversely proportional to $\mu_{w}$, meaning that increasing the outer viscosity favors the abrupt velocity jump. This dependence leads to counter-intuitive results when viscous glycerol is added to the outer aqueous solution (50\% in volume of glycerol, as detailed in the Supplementary). In Fig. 4 we compare the falling velocity of droplets in solutions of viscosity $\mu_{w}=1$ and $8 \mathrm{mPa}$.s. In the case of smooth surfaces, the droplets move faster in the solution of lower viscosity, in agreement with Eq. 2 and common intuition. However, the case of rough surfaces is more surprising. For high confinement, the velocity is low and does not display

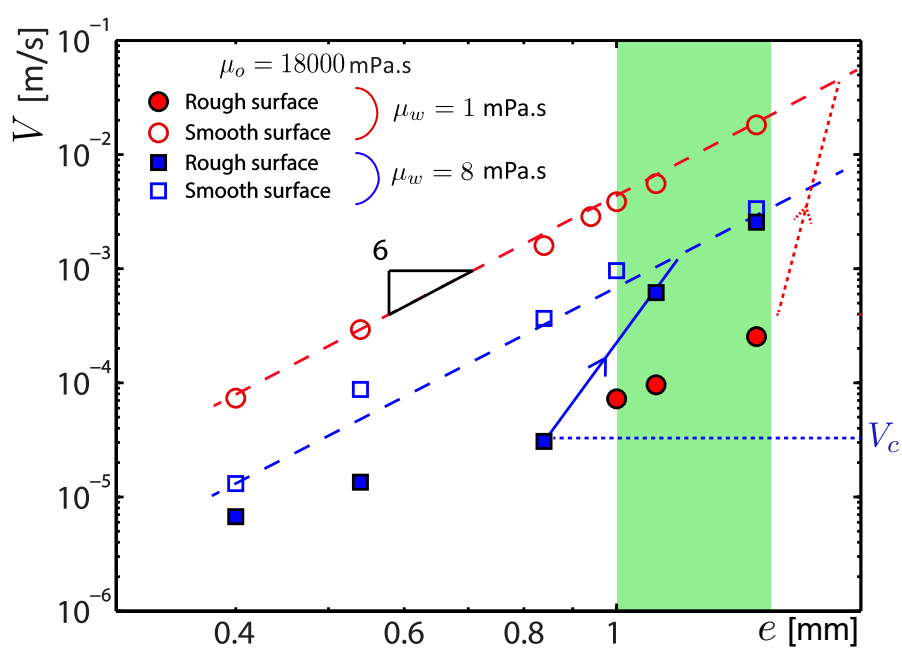

FIG. 4: $V$ vs. $e$ for drops of $\mu_{o}=18000 \mathrm{mPa} . \mathrm{s}$ in smooth and rough confinements represented, respectively, by empty and full symbols. The blue squares correspond to droplets of $\mu_{o}=18000 \mathrm{mPa}$.s advancing in vertical Hele-Shaw cells filled with a viscous water-glycerol mixture $\left(\mu_{w}=8 \mathrm{mPa} . \mathrm{s}\right)$. The red circles to droplets of $\mu_{o}=18000 \mathrm{mPa} . \mathrm{s}$ advancing in Hele-Shaw cells filled with water $\left(\mu_{w}=1 \mathrm{mPa} . \mathrm{s}\right)$ inclined by an angle of $30^{\circ}$ with respect to the horizontal. This inclination is realized to counteract the change of density of the aqueous solution $\rho_{w}$ due to the addition of dense glycerol and to guarantee a similar driving force between both experiments (see the Supplementary for more details). In the greencolored region, much larger velocities are counter-intuitively obtained for drops evolving in a more viscous environment $\left(\mu_{w}=8 \mathrm{mPa} . \mathrm{s}\right.$, see also the Supplemental Movie 2). The red dashed arrow represents the velocity jump which would occur if higher gap thickness $e$ could be tested.

a strong dependence on $\mu_{w}$. The jump appears first with the viscous solution, leading to a sharp increase of the velocity as expected from Eq. 3. Within a certain range of confinement (green region in Fig. 4), droplets thus move faster when surrounded by a more viscous solution (see also the Supplemental Movie 2). Provided higher gap thicknesses could be tested, one would also expect the data for $\mu_{w}=1 \mathrm{mPa}$.s to exhibit an abrupt velocity jump (dashed arrows in Fig. 4), and the intuitive behavior would be restored: the more viscous the outer phase, the slower the drops.

This Letter illustrates the intricate dissipation mechanisms acting at the vicinity of rough surfaces. Below a critical velocity, roughness decreases the friction at the front meniscus of the drop. As an illustration, we show in the Supplementary that buoyant confined air bubbles tend to rise faster in textured Hele-Shaw cells than between smooth walls. In this opposite configuration, the outer fluid is more viscous than the inner air and the motion is mainly resisted by friction in the vicinity of the peripheral menisci $[33,34]$. The higher mobility observed at small $\mathrm{Ca}$ highlights role of roughness in reduc- 
ing friction. In the case of viscous drops, the impact of roughness is dramatically different. The reduced viscous friction at the front of the drop hinders the deformation of the interface and the formation of an efficient lubricating film. This results in a corrugated interface (Fig. 3a) and in the localization of viscous friction in the viscous drop. We finally showed that increasing the viscosity of the outer fluid, the viscous friction at the periphery is increased and a thick lubricating layer is restored, therefore leading to faster drops.

Depending on the inner-to-outer viscosity ratio $\mu_{o} / \mu_{w}$, we showed that the apparent roughness-induced reduction of friction in the vicinity of the meniscus can accelerate air bubbles or, as a paradox, substantially slow down highly viscous droplets. A direct observation of the structure of the lubricating film would enable to accurately describe the configurations qualitatively represented in Fig 3a, using for instance Reflection Interference Contrast Microscopy (RICM) [11, 17] or confocal microscopy [35].

Although we focused on a sedimentation experiment, the observed results are fully applicable to configurations where drops are entrained by a surrounding fluid in a confined environment, such as microfluidic channels $[7,8]$ or porous media [6]. From a practical point of view, controlling the friction of viscous droplets through a designed texture might constitute an efficient tool to probe the rheological properties of droplets, vesicles or biological cells, and possibly achieve specific sorting.

\section{Acknowledgements}

The authors thank K. Jaafar for his initial contribution to the project, and acknowledge funding from Total S.A. This work has received the support of Institut PierreGilles de Gennes, "Investissements d'avenir", program ANR-10-EQPX-34.

* Electronic address: ludovic.keiser@espci.org

$\dagger$ Electronic address: etienne.reyssat@espci.fr

[1] T. C. Ransohoff, P. A. Gauglitz, and C. J. Radke. Snapoff of gas bubbles in smoothly constricted noncircular capillaries. AIChE J., 33(5):753-765, 1987.

[2] P. G. Saffman and G. I. Taylor. The penetration of a fluid into a porous medium or Hele-Shaw cell containing a more viscous liquid. Proc. R. Soc. Lond. A., 245(1242):312-329, 1958.

[3] Talal T. Al-Housseiny, Peichun A Tsai, and Howard A Stone. Control of interfacial instabilities using flow geometry. Nat. Phys., 8(10):747-750, 2012.

[4] C. Odier, B. Levaché, E. Santanach-Carreras, and D. Bartolo. Forced Imbibition in Porous Media: A Fourfold Scenario. Phys. Rev. Lett., 119:208005, 2017.
[5] L. Keiser, R. Herbaut, J. Bico, and É. Reyssat. Washing wedges: capillary instability in a gradient of confinement. J. Fluid. Mech., 790:619-633, 2016.

[6] A. Z. Zinchenko and R. H. Davis. Motion of Deformable Drops Through Porous Media. Ann. Rev. Fluid Mech., 49(1):71-90, 2017.

[7] C. Baroud, F. Gallaire, and R. Dangla. Dynamics of microfluidic droplets. Lab on a Chip, 10:2032-2045, 2010.

[8] R. Seemann, M. Brinkmann, T. Pfohl, and S. Herminghaus. Droplet based microfluidics. Rep. Prog. Phys., $75: 16601,2012$.

[9] A. de Lózar, A. L. Hazel, and A. Juel. Scaling Properties of Coating Flows in Rectangular Channels. Phys. Rev. Lett., 99(23):234501, 2007.

[10] S. Jakiela, P. M. Korczyk, S. Makulska, O. Cybulski, and P. Garstecki. Discontinuous Transition in a Laminar Fluid Flow: A Change of Flow Topology inside a Droplet Moving in a Micron-Size Channel. Phys. Rev. Lett., 108(13):134501, 2012.

[11] A. Huerre, O. Theodoly, A. M. Leshansky, M.-P. Valignat, I. Cantat, and M.-C. Jullien. Droplets in Microchannels: Dynamical Properties of the Lubrication Film. Phys. Rev. Lett., 115(6):064501, 2015.

[12] L. Zhu and F. Gallaire. A pancake droplet translating in a Hele-Shaw cell: lubrication film and flow field. J. Fluid. Mech., 2016.

[13] Y. Ling, J.-M. Fullana, S. Popinet, and C. Josserand. Droplet migration in a Hele-Shaw cell: Effect of the lubrication film on the droplet dynamics. Phys. Fluids, 28(6):062001, 2016.

[14] M. Yahashi, N. Kimoto, and K. Okumura. Scaling crossover in thin-film drag dynamics of fluid drops in the Hele-Shaw cell. Sci. Rep., 6(1):31395, 2016.

[15] G. Balestra, L. Zhu, and F. Gallaire. A viscous droplet in a capillary tube: from Bretherton's theory to empirical models. Microfluid. Nanofluid, 22(6):67, 2018.

[16] L. Keiser, K. Jaafar, J. Bico, and É. Reyssat. Dynamics of non-wetting drops confined in a Hele-Shaw cell. J. Fluid Mech., 845:245-262, 2018.

[17] B. Reichert, A. Huerre, O. Théodoly, M.-P. Valignat, I. Cantat, and M.-C. Jullien. Topography of the lubrication film under a pancake droplet traveling in a HeleShaw cell. J. Fluid Mech., 850:708-732, 2018.

[18] O. I. Vinogradova. Slippage of water over hydrophobic surfaces. Int. J. Miner. Process., 56(1-4):31-60, apr 1999.

[19] É. Lauga and H.A. Stone. Effective slip in pressure-driven Stokes flow. J. Fluid. Mech., 489:55-77, 2003.

[20] C.-H. Choi and C.-J. Kim. Large Slip of Aqueous Liquid Flow over a Nanoengineered Superhydrophobic Surface. Phys. Rev. Lett., 96(6):066001, 2006.

[21] C. Ybert, C. Barentin, C. Cottin-Bizonne, P. Joseph, and L. Bocquet. Achieving large slip with superhydrophobic surfaces: Scaling laws for generic geometries. Phys. Fluids, 19(12):123601, 2007.

[22] J. P. Rothstein. Slip on Superhydrophobic Surfaces. Ann. Rev. Fluid Mech., 42(1):89-109, 2010.

[23] D. Schäffel, K. Koynov, D. Vollmer, H. J. Butt, and C. Schönecker. Local Flow Field and Slip Length of Superhydrophobic Surfaces. Phys. Rev. Lett., 116(13):134501, 2016.

[24] J. D. Smith, R. Dhiman, S. Anand, E. Reza-Garduno, R. E. Cohen, G. H. McKinley, and K. K. Varanasi. Droplet mobility on lubricant-impregnated surfaces. Soft 
Matt., 9(6):1772-1780, 2013.

[25] D. Daniel, J. Timonen, R. Li, S. Velling, and J. Aizenberg. Oleoplaning droplets on lubricated surfaces. Nat. Phys., 13(10):1020-1025, 2017.

[26] A. Keiser, L. Keiser, C. Clanet, and D. Quéré. Drop friction on liquid-infused materials. Soft Matt., 13(39):69816987, 2017.

[27] F. P. Bretherton. The motion of long bubbles in tubes. J. Fluid. Mech., 10(02):166-188, 1961.

[28] J. Seiwert, C. Clanet, and D. Quéré. Coating of a textured solid. J. Fluid. Mech., 669:55-63, 2011.

[29] J. N. Israelachvili. Intermolecular and surface forces. 3rd edn. Elsevier, 2011.

[30] D. Einzel, P. Panzer, and M. Liu. Boundary condition for fluid flow: Curved or rough surfaces. Phys. Rev. Lett., 64(19):2269-2272, 1990.
[31] Y.-C. Liao, Y.-C. Li, and H.-H. Wei. Drastic Changes in Interfacial Hydrodynamics due to Wall Slippage: SlipIntensified Film Thinning, Drop Spreading, and Capillary Instability. Phys. Rev. Lett., 111(13):136001, 2013.

[32] Y.-C. Li, Y.-C. Liao, T.-C. Wen, and H.-H. Wei. Breakdown of the Bretherton law due to wall slippage. J. Fluid Mech., 741:200-227, 2014.

[33] I. Cantat. Liquid meniscus friction on a wet plate: Bubbles, lamellae, and foams. Phys. Fluids, 25:031303, 2013.

[34] É. Reyssat. Drops and bubbles in wedges. J. Fluid Mech., 748:641-662, 2014.

[35] F. Schellenberger, J. Xie, N. Encinas, A. Hardy, M. Klapper, P. Papadopoulos, H.-J. Butt, and D. Vollmer. Direct observation of drops on slippery lubricant-infused surfaces. Soft Matt., 11(38):7617-7626, 2015. 